\title{
Source Code Security Analysis Tool Test Plan Version 1.1
}

\author{
Michael Koo \\ Romain Gaucher \\ Charline Cleraux \\ Jenise Reyes Rodriguez
}

Software and Systems Division Information Technology Laboratory National Institute of Standards and Technology Gaithersburg, MD 20899

July 2011

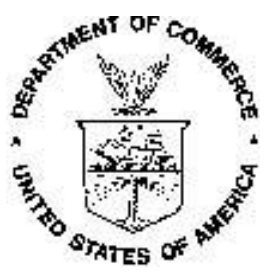

U.S. Department of Commerce Rebecca M. Blank, Acting Secretary 


\section{Abstract}

This document provides a set of metrics, including test suites and methods, to determine how well a particular source code security analysis tool conforms to the requirements specified in Source Code Security Analysis Tool Functional Specification Version 1.0 [SCA]. Each relevant programming language in [SCA] has a corresponding set of test suites. The test suites are intended to be used by tool developers and tool users alike to increase their level of confidence in product quality. Each test suite consists of test cases that are designed to evaluate against various requirements of [SCA], including mandatory features and optional features. Each test case contains a test description, a description of the weakness contained in the test case, the expected result and the test code. Detailed information on the test case, such as start parameters, procedures for executing the test file and the test file itself can be retrieved from the SAMATE Reference Dataset (SRD) at http://samate.nist.gov/SRD/.

As this document evolves, new versions will be posted to the web site at http://samate.nist.gov/index.php/Source_Code_Security_Analysis.html.

\section{Keywords}

Source code security analysis tool; test plan; test methodology; test suite.

\section{Changes to this version}

This version 1.1 updates version 1.0 by modifying $\mathrm{C}$ and $\mathrm{C}++$ test suites and adding Java test suites. 


\section{Table of Contents}

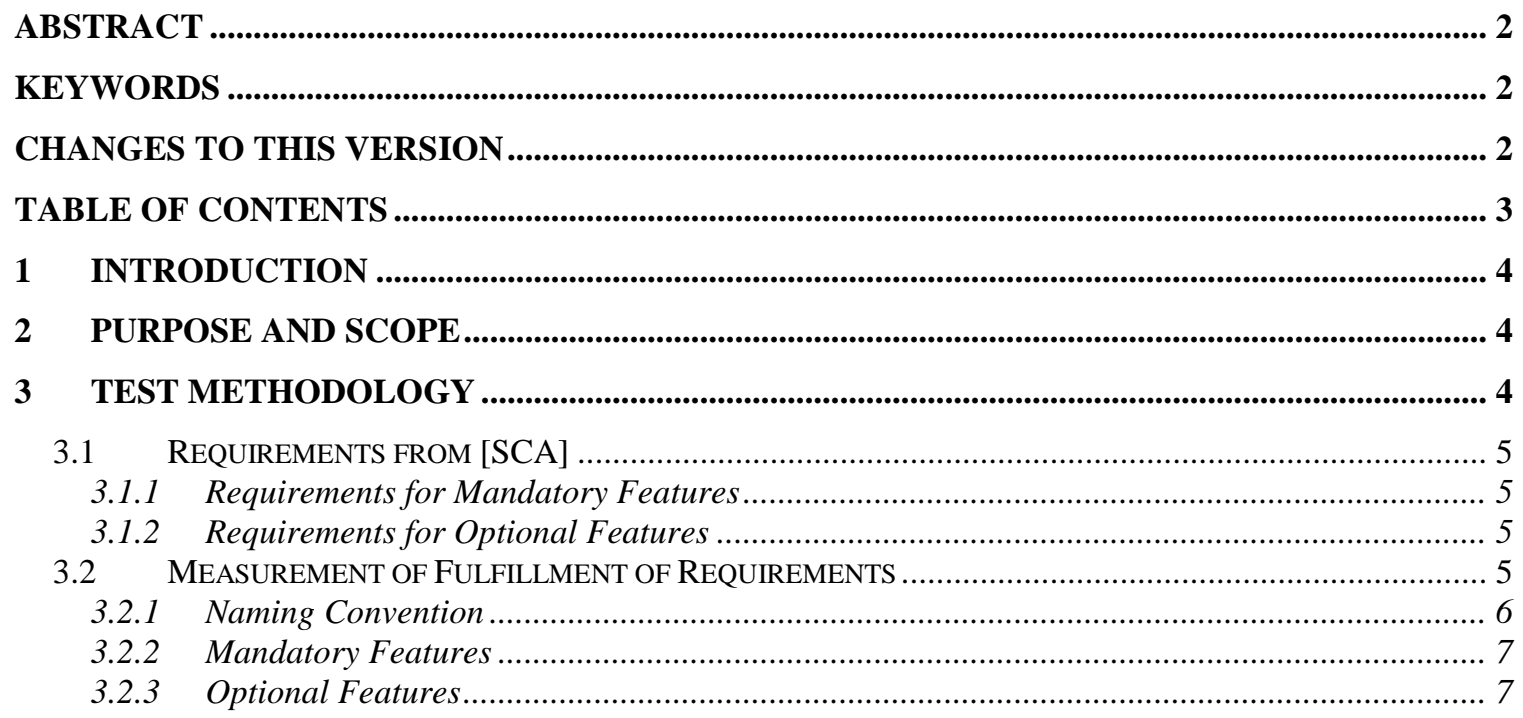

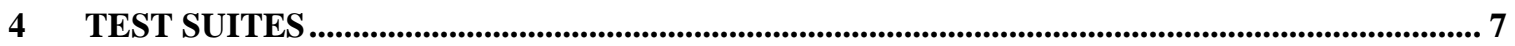

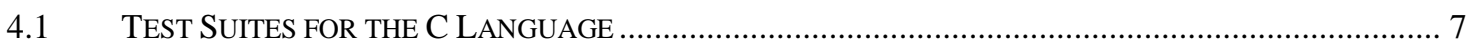

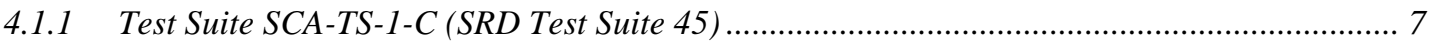

4.1.2 Test Suite SCA-TS-2-C (SRD Test Suite 46) …….........................................................

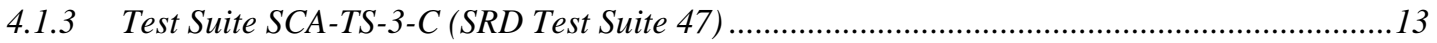

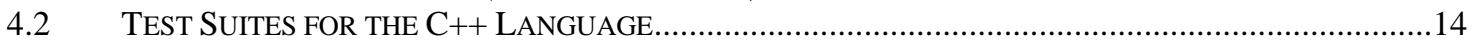

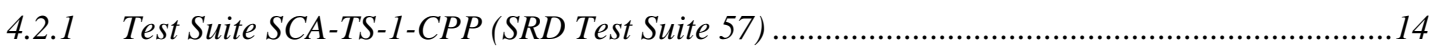

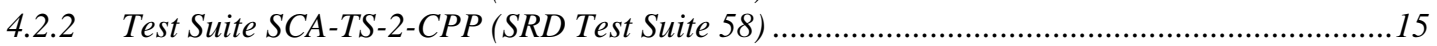

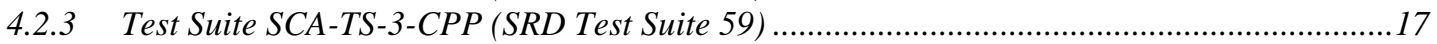

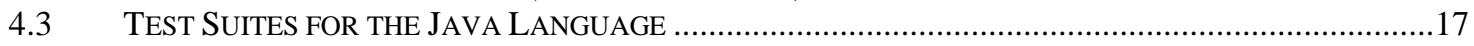

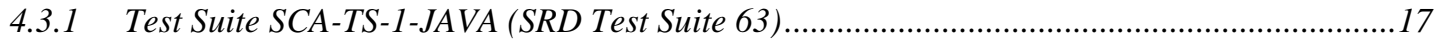

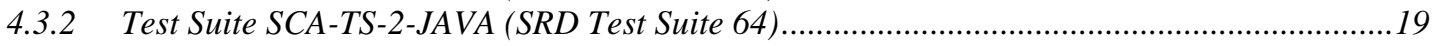

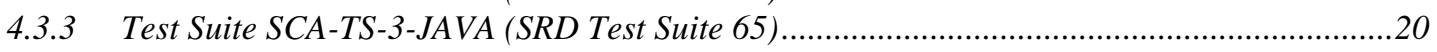

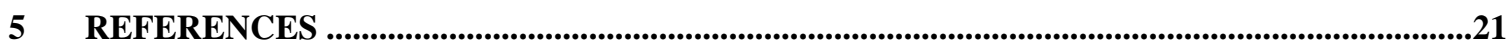




\section{Introduction}

There is a critical need in information technology to ensure that software assurance tools produce accurate, repeatable and objective results. The Software Assurance Metrics and Tool Evaluation (SAMATE) project at the National Institute of Standards and Technology (NIST) ${ }^{1}$ is working to establish a methodology for measuring the functionality and capability of software assurance tools by developing functional specifications, test procedures, test criteria, and test suites. The results provide the information necessary for the toolmakers to improve tools, for users to make informed choices about acquiring and using software assurance tools, and for interested parties to understand the tools' capabilities.

This document updates a test plan, including the test methodology and test suites, for Source Code Security Analysis Tools that examine program source code to detect and report weaknesses that can lead to security vulnerabilities. Other static analysis tools, for examples tools that scan bytecode or binary code, are not covered.

\section{Purpose and Scope}

This document, along with some specific test suites in the SAMATE Reference Dataset (SRD) [SRD], provides a means to test the functionality and capability of a Source Code Security Analysis Tool based on the requirements asserted in Source Code Security Analysis Tool Functional Specification Version 1.0 [SCA].

The test plan is generic in that it can be applied to any programming language. However, each language will have its own specific set of test suites for measuring the tool.

The test methodology described in this document focuses only on the functional specification in [SCA]. Testing the performance robustness, scalability, usability, etc. of a source code security analysis tool is outside of the scope of this document.

\section{Test Methodology}

\footnotetext{
1 This project is sponsored by the U.S. Department of Homeland Security (DHS) National Cyber Security Division and NIST. Detailed information about this project is described at http://samate.nist.gov/.
} 


\subsection{Requirements from [SCA]}

\subsubsection{Requirements for Mandatory Features}

To meet a minimum level of capability, a source code security analysis tool or set of tools must be able to accomplish the tasks described below. The tool(s) shall:

SCSA-RM-1: Identify all of the classes of weaknesses listed in Annex A of [SCA].

SCSA-RM-2: Textually report any weaknesses that it identifies.

SCSA-RM-3: For any identified weaknesses in classes listed in Annex A of [SCA], report the class using a semantically equivalent name.

SCSA-RM-4: Report the location of any weaknesses by providing the directory path, file name and line number.

SCSA-RM-5: Identify weaknesses despite the presence of the coding complexities listed in Annex B of [SCA].

SCSA-RM-6: Have an acceptably low false positive rate.

\subsubsection{Requirements for Optional Features}

The following requirements apply to optional tool features. If the tool supports an optional feature, then the requirement for that feature applies, and the tool can be tested against it. A specific tool might optionally provide none, some, or all of the features described by these requirements. Optionally, the tool(s) shall:

SCSA-RO-1: Produce an XML-formatted report.

SCSA-RO-2: Not report a weakness instance that has been suppressed.

SCSA-RO-3: Use the Common Weakness Enumeration [CWE] name of the weakness class it reports.

\subsection{Measurement of Fulfillment of Requirements}

In general, measuring source code security analysis tool takes three steps:

1. Preparation: Set up testing environment, study functionalities of the tool, become familiar with the material in Annex A of [SCA], review test suites outlined in this test plan, etc. Specific examples of tasks that may be part of this step include:

- install tool

- understand the capacity of the tool

- establish the corresponding relationship between weaknesses that the tool can detect and the weakness listed in Annex A of [SCA]

- choose appropriate test suites, (see Section 4).

2. Run tool on test cases in test suites - determine if results are as expected. 


\section{Summarize results.}

This test plan includes 9 (nine) test suites. Each test suite evaluates the source code security tool for one or multiple requirement specified in Sections 3.1.1 and 3.1.2. Each test suite consists of a set of test cases in one of the languages, i.e., C, C++, or Java. A test case demonstrates a weakness in a specific code construct; or a test case is a fixed version of code that has the weakness removed to measure the capability of the tool to avoid generating false positives.

The expected result of a test case will be a report of weakness, if a weakness is seeded in the code; or it will not report the weakness, if a weakness is not seeded or reporting the weakness is suppressed. If a weakness is reported, the expected result must be a weakness from classes listed in Annex A of [SCA], or a weakness from that class reported using a semantically equivalent name. The report must also include the location of the weakness.

\subsubsection{Naming Convention}

Annex A of [SCA] covers three computer languages, C, C++ and Java. Each computer language has three corresponding test suites. Test suite SCA-TS-1-name, where name is the name of the language, consists of test cases in that language. For instance, SCA-TS-1-CPP is for the C++ language and SCA-TS-1-Java is for Java. Test suite SCA-TS-1 tests features that are described in requirements SCARM-1 through SCA-RM-5. It can also test requirements SCA-RO-1 and SCA-RO-3. Test suite SCA-TS-2-name tests requirement SCA-RM-6, false alarms or false positives. Test suite SCA-TS-3 tests requirement SCA-RO-2, suppression of warnings.

Dependent on the language and requirements to be tested, download the appropriate test suite from the SRD, see Section 4.

Since there may be up to several hundred individual test results to check, we encourage users to write a harness for their own needs. The "More Downloads" section of the SRD provides sample scripts. All the tests could be run, and results saved, then the determination made if the results are as expected. Alternatively the determination could be made as each test is run.

Note that the results from running the tool on test suite SCA-TS-1 are used for many requirements. The results of SCA-TS-2 and SCA-TS-3 are used for one requirement each. 


\subsubsection{Mandatory Features}

- To determine if SCA-RM-1 is met from the results of SCA-TS-1 ... To determine if SCA-RM-2 is met from the results of SCA-TS-1 ... To determine if SCA-RM-3 is met from the results of SCA-TS-1 ... To determine if SCA-RM-4 is met from the results of SCA-TS-1 ... To determine if SCA-RM-5 is met from the results of SCA-TS-1 ...

For each test case, the tool under test is expected to generate a report that identifies the seeded weakness with a name semantically equivalent to one of those in Annex A of [SCA] and its location (e.g., the path name and line number(s) of the weakness).

- To determine what to report for SCA-RM-6 from the results of SCA-TS-2 ... For each test case, the original seeded weakness is fixed or removed. The tool under test should not report any weakness. If it does, practical considerations require the false positive rate [Fleiss] to be acceptably low for the domain.

\subsubsection{Optional Features}

- To test requirement SCA-RO-1, turn on that feature if necessary and then run test suite SCA-TS-1 for the appropriate language. The tool under test should generate a report as described in 3.2.2 in the appropriate XML-format.

- To test requirement SCA-RO-2, run test suite SCA-TS-3 first to prove the tool can identify the weaknesses. Then run SCA-TS-3 again after the weaknesses described in Annex A of [SCA] are suppressed in the tool under test. The tool should generate no reports for any weakness that is suppressed.

- To test requirement SCA-RO-3, run test suite SCA-TS-1 for the appropriate language. For each test case, the tool under test is expected to generate a report that identifies the incorporated weakness with the correct CWE name.

\section{Test Suites}

A test suite is a collection of test cases explicitly selected for a special purpose. Each test case is an atomic program that tests a specific tool functionality required by [SCA]. Test suites and their test cases are stored in the SRD [SRD]. Each SRD test case entry provides a test file, a description of the test case, the CWE classification of the incorporated weakness, any type(s) of code complexity present, the location of the weakness, and other test case related information. This test plan includes test suites for $\mathrm{C}$, $\mathrm{C}++$ and Java languages.

\subsection{Test Suites for the C Language}

\subsubsection{Test Suite SCA-TS-1-C (SRD Test Suite 45)} http://samate.nist.gov/SRD/view.php?tsID=45 
This test suite tests the capability of a source code security analysis tool's handling of weaknesses in the $\mathrm{C}$ language. It covers the source code weaknesses in $\mathrm{C}$ listed in Annex A of Source Code Security Analysis Tool Functional Specification [SCA].

\begin{tabular}{|c|c|c|c|c|}
\hline $\begin{array}{l}\text { Source Code } \\
\text { Weakness }\end{array}$ & $\begin{array}{l}\text { CWE } \\
\text { ID }\end{array}$ & Code Complexity & $\begin{array}{l}\text { SRD } \\
\text { Test } \\
\text { case ID }\end{array}$ & Remark \\
\hline \multirow[t]{5}{*}{ Basic XSS } & 80 & Basic & 1794 & \\
\hline & & Scope & 1781 & \\
\hline & & Address alias level & 1919 & \\
\hline & & Container & 1921 & \\
\hline & & Loop complexity & 2198 & \\
\hline \multirow{4}{*}{$\begin{array}{l}\text { Resource } \\
\text { Injection }\end{array}$} & 99 & Basic & 1897 & \\
\hline & & Scope & 1901 & \\
\hline & & Address alias level & 1895 & \\
\hline & & Container & 1899 & \\
\hline \multirow[t]{4}{*}{$\begin{array}{l}\text { OS Command } \\
\text { Injection }\end{array}$} & 78 & Basic & 111 & $\begin{array}{l}\text { Test function } \\
\text { 'system()'. }\end{array}$ \\
\hline & & Scope & 1885 & \\
\hline & & Local control flow & 1881 & \\
\hline & & Loop structure & 1883 & \\
\hline \multirow[t]{3}{*}{ SQL Injection } & 89 & Basic & 1796 & \\
\hline & & Array index complexity & 1798 & \\
\hline & & Scope & 1800 & \\
\hline \multirow[t]{9}{*}{ Stack Overflow } & 121 & Basic & 2009 & \\
\hline & & Array index complexity & 1544 & \\
\hline & & Scope & 1548 & \\
\hline & & Basic & 1563 & Test function gets() \\
\hline & & Basic & 1565 & Test function fgets() \\
\hline & & Array index complexity & 1751 & \\
\hline & & $\begin{array}{l}\text { Array length/limit } \\
\text { complexity }\end{array}$ & 1905 & \\
\hline & & Index alias level & 1907 & \\
\hline & & Loop Structure & 1909 & \\
\hline \multirow[t]{4}{*}{ Heap Overflow } & 122 & Basic & 1611 & \\
\hline & & Scope & 1612 & \\
\hline & & $\begin{array}{l}\text { Array address } \\
\text { complexity }\end{array}$ & 1843 & \\
\hline & & Array index complexity & 1845 & \\
\hline Format string & 134 & Basic & 10 & \\
\hline
\end{tabular}




\begin{tabular}{|c|c|c|c|c|}
\hline \multicolumn{5}{|l|}{ vulnerability } \\
\hline & & Address alias level & 92 & \\
\hline & & Scope & 93 & \\
\hline & & Container & 1831 & \\
\hline & & Local control flow & 1833 & \\
\hline \multirow{5}{*}{$\begin{array}{l}\text { Improper Null } \\
\text { Termination }\end{array}$} & 170 & Basic & 1849 & \\
\hline & & Taint & 1857 & \\
\hline & & Buffer address type & 2010 & \\
\hline & & Container & 1854 & \\
\hline & & Address alias level & 1850 & \\
\hline Heap Inspection & 244 & Basic & 1737 & \\
\hline \multirow{5}{*}{$\begin{array}{l}\text { Often Misused: } \\
\text { String } \\
\text { Management }\end{array}$} & 251 & Basic & 1865 & \\
\hline & & Taint & 1873 & \\
\hline & & Scope & 1871 & \\
\hline & & Address alias level & 1867 & \\
\hline & & Container & 1869 & \\
\hline \multirow{5}{*}{$\begin{array}{l}\text { Hard-coded } \\
\text { Password }\end{array}$} & 259 & Basic & 1810 & \\
\hline & & Local control flow & 1839 & \\
\hline & & Loop structure & 1841 & \\
\hline & & Container & 1837 & \\
\hline & & $\begin{array}{l}\text { Array Index } \\
\text { Complexity }\end{array}$ & 1835 & \\
\hline \multirow{3}{*}{$\begin{array}{l}\text { Time-of-check } \\
\text { Time-of-use race } \\
\text { condition }\end{array}$} & 367 & Basic & 102 & \\
\hline & & Basic & 1806 & \\
\hline & & Local Control Flow & 1808 & \\
\hline $\begin{array}{l}\text { Unchecked Error } \\
\text { Condition }\end{array}$ & 391 & Basic & 1928 & \\
\hline \multirow[t]{2}{*}{ Memory leak } & 401 & Basic & 1585 & \\
\hline & & Scope & 1588 & \\
\hline $\begin{array}{l}\text { Unrestricted } \\
\text { Critical Resource } \\
\text { Lock }\end{array}$ & 412 & Basic & 2109 & \\
\hline \multirow[t]{3}{*}{ Double Free } & 415 & Basic & 2199 & \\
\hline & & Buffer address type & 99 & \\
\hline & & Loop structure & 1829 & \\
\hline
\end{tabular}




\begin{tabular}{|l|l|l|l|l|l|}
\hline & & Local control flow & 1827 & \\
\hline Use After Free & 416 & $\begin{array}{l}\text { Scope } \\
\text { Basic }\end{array}$ & 1590 & \\
& & Scope & 2200 & \\
Container & 2201 & \\
\hline & & $\begin{array}{l}\text { Buffer address type } \\
\text { Data type }\end{array}$ & 2202 & \\
\hline $\begin{array}{l}\text { Uninitialized } \\
\text { variable }\end{array}$ & 457 & 2019 & Data type is integer \\
\hline & & Data type & 2003 & Data type is int * \\
\hline $\begin{array}{l}\text { Unintentional } \\
\text { pointer scaling }\end{array}$ & 468 & Loop Structure & 1757 & \\
\hline Null Dereference & 476 & Basic & 1782 & \\
\hline & & Address alias level & 1875 & \\
\hline & & Local control flow & 1877 & \\
\hline $\begin{array}{l}\text { Leftover Debug } \\
\text { Code }\end{array}$ & 489 & Basic & 1879 & \\
\hline
\end{tabular}

\subsubsection{Test Suite SCA-TS-2-C (SRD Test Suite 46)} http://samate.nist.gov/SRD/view.php?tsID=46

This test suite can be used to assess the false positive ratio of the tool under test for $\mathrm{C}$ applications.

\begin{tabular}{|l|l|l|l|l|}
\hline $\begin{array}{l}\text { Source Code } \\
\text { Weakness }\end{array}$ & $\begin{array}{l}\text { CWE } \\
\text { ID }\end{array}$ & Code Complexity & $\begin{array}{l}\text { SRD } \\
\text { Test case } \\
\text { ID }\end{array}$ & Remark \\
\hline Basic XSS & 80 & Basic & 1795 & \\
\hline & & Scope & 1924 & \\
\hline & & $\begin{array}{l}\text { Address alias level } \\
\text { Container }\end{array}$ & 1920 & \\
\hline & & Loop complexity & 2204 & \\
\hline $\begin{array}{l}\text { Resource } \\
\text { Injection }\end{array}$ & 99 & Basic & 1898 & \\
\hline & & Address alias level & 1896 & \\
\hline & & Container & 1900 & \\
\hline OS Command & 78 & Scope & 1902 & \\
\hline Injection & & Basic & 2139 & $\begin{array}{l}\text { Test function } \\
\text { 'system()'. }\end{array}$ \\
\hline & & scope & 2138 & \\
\hline
\end{tabular}




\begin{tabular}{|c|c|c|c|c|}
\hline & & Local control flow & 2136 & \\
\hline & & loop structure & 2137 & \\
\hline \multirow[t]{4}{*}{ SQL Injection } & 89 & Basic & 1797 & \\
\hline & & $\begin{array}{l}\text { Array index } \\
\text { complexity }\end{array}$ & 1799 & \\
\hline & & Scope & 1801 & \\
\hline & & Loop structure & 1930 & \\
\hline \multirow[t]{8}{*}{ Stack Overflow } & 121 & Basic & 1547 & \\
\hline & & Array index complexity & 1545 & \\
\hline & & Scope & 1549 & \\
\hline & & Basic & 1566 & \\
\hline & & $\begin{array}{l}\text { Array length/limit } \\
\text { complexity }\end{array}$ & 1906 & \\
\hline & & Basic & 1602 & \\
\hline & & Index alias level & 1908 & \\
\hline & & Loop Structure & 1910 & \\
\hline \multirow[t]{6}{*}{ Heap Overflow } & 122 & Basic & 2134 & \\
\hline & & Scope & 1615 & \\
\hline & & $\begin{array}{l}\text { Array index } \\
\text { complexity }\end{array}$ & 1844 & \\
\hline & & Memory location & 1848 & \\
\hline & & Array index complexity & 1574 & \\
\hline & & Scope & 1613 & \\
\hline \multirow{5}{*}{$\begin{array}{l}\text { Format string } \\
\text { vulnerability }\end{array}$} & 134 & Scope & 1562 & \\
\hline & & Address alias level & 1560 & \\
\hline & & Local control flow & 1834 & \\
\hline & & Container & 1832 & \\
\hline & & Scope & 1556 & \\
\hline \multirow{4}{*}{$\begin{array}{l}\text { Improper Null } \\
\text { Termination }\end{array}$} & 170 & Basic & 1856 & \\
\hline & & Taint & 1858 & \\
\hline & & Buffer address type & 2012 & \\
\hline & & Container & 1855 & \\
\hline \multirow{5}{*}{$\begin{array}{l}\text { Often Misused: } \\
\text { String } \\
\text { Management }\end{array}$} & 251 & Basic & 1866 & \\
\hline & & Taint & 1874 & \\
\hline & & Scope & 1872 & \\
\hline & & Address alias level & 1868 & \\
\hline & & Container & 1870 & \\
\hline
\end{tabular}




\begin{tabular}{|c|c|c|c|}
\hline \multirow{4}{*}{$\begin{array}{l}\text { Hard-coded } \\
\text { Password }\end{array}$} & 259 & Basic & 2131 \\
\hline & & Local control flow & 2132 \\
\hline & & Loop structure & 2133 \\
\hline & & $\begin{array}{l}\text { Array Address } \\
\text { Complexity }\end{array}$ & 2130 \\
\hline \multirow{2}{*}{$\begin{array}{l}\text { Time-of-check } \\
\text { Time-of-use race } \\
\text { condition }\end{array}$} & 367 & Basic & 1892 \\
\hline & & Local Control Flow & 1894 \\
\hline $\begin{array}{l}\text { Unchecked Error } \\
\text { Condition }\end{array}$ & 391 & Basic & 1929 \\
\hline \multirow[t]{5}{*}{ Memory leak } & 401 & Basic & 1933 \\
\hline & & Scope & 1586 \\
\hline & & Address alias level & 1589 \\
\hline & & Container & 1925 \\
\hline & & Loop structure & 1926 \\
\hline $\begin{array}{l}\text { Unrestricted } \\
\text { Critical Resource } \\
\text { Lock }\end{array}$ & 412 & Basic & 2205 \\
\hline \multirow[t]{4}{*}{ Double Free } & 415 & Basic & 2271 \\
\hline & & Loop structure & 1830 \\
\hline & & local control flow & 1828 \\
\hline & & Scope & 1591 \\
\hline \multirow[t]{4}{*}{ Use After Free } & 416 & Scope & 2269 \\
\hline & & Address alias level & 2270 \\
\hline & & Container & 2135 \\
\hline & & Buffer address type & 1914 \\
\hline $\begin{array}{l}\text { Uninitialized } \\
\text { variable }\end{array}$ & 457 & Basic & 2186 \\
\hline $\begin{array}{l}\text { Unintentional } \\
\text { pointer scaling }\end{array}$ & 468 & Data type & 1927 \\
\hline \multirow[t]{4}{*}{ Null Dereference } & 476 & Basic & 2195 \\
\hline & & Scope & 1880 \\
\hline & & Address alias level & 1876 \\
\hline & & Local control flow & 2194 \\
\hline $\begin{array}{l}\text { Leftover Debug } \\
\text { Code }\end{array}$ & 489 & Basic & 1862 \\
\hline
\end{tabular}




\subsubsection{Test Suite SCA-TS-3-C (SRD Test Suite 47)}

http://samate.nist.gov/SRD/view.php?tsID=47

This test suite can be used to examine whether the tool generates weakness reports after reporting of each weakness has been suppressed for $\mathrm{C}$ applications.

\begin{tabular}{|c|c|c|c|c|}
\hline $\begin{array}{l}\text { Source Code } \\
\text { Weakness }\end{array}$ & $\begin{array}{l}\text { CWE } \\
\text { ID }\end{array}$ & Code Complexity & $\begin{array}{l}\text { SRD } \\
\text { Test } \\
\text { case ID }\end{array}$ & Remark \\
\hline Basic XSS & 80 & Basic & 1794 & \\
\hline $\begin{array}{l}\text { Resource } \\
\text { Injection }\end{array}$ & 99 & Basic & 1897 & \\
\hline $\begin{array}{l}\text { OS Command } \\
\text { Injection }\end{array}$ & 78 & Basic & 1885 & $\begin{array}{l}\text { Test function } \\
\text { 'system()'. }\end{array}$ \\
\hline SQL Injection & 89 & Basic & 1796 & \\
\hline Stack Overflow & 121 & Basic & 1563 & \\
\hline Heap Overflow & 122 & Basic & 1611 & \\
\hline $\begin{array}{l}\text { Format string } \\
\text { vulnerability }\end{array}$ & 134 & Basic & 10 & \\
\hline $\begin{array}{l}\text { Improper Null } \\
\text { Termination }\end{array}$ & 170 & Basic & 1849 & \\
\hline Heap Inspection & 244 & Basic & 1737 & \\
\hline $\begin{array}{l}\text { Often Misused: } \\
\text { String } \\
\text { Management }\end{array}$ & 251 & Basic & 1865 & \\
\hline $\begin{array}{l}\text { Hard-coded } \\
\text { Password }\end{array}$ & 259 & Basic & 1810 & \\
\hline $\begin{array}{l}\text { Time-of-check } \\
\text { Time-of-use race } \\
\text { condition }\end{array}$ & 367 & Basic & 102 & \\
\hline $\begin{array}{l}\text { Unchecked Error } \\
\text { Condition }\end{array}$ & 391 & Basic & 1928 & \\
\hline Memory leak & 401 & Basic & 1585 & \\
\hline $\begin{array}{l}\text { Unrestricted } \\
\text { Critical Resource } \\
\text { Lock }\end{array}$ & 412 & Basic & 2109 & \\
\hline Double Free & 415 & Basic & 2199 & \\
\hline Use After Free & 416 & Basic & 2200 & \\
\hline $\begin{array}{l}\text { Uninitialized } \\
\text { variable }\end{array}$ & 457 & Data type & 2019 & Data type is integer \\
\hline $\begin{array}{l}\text { Unintentional } \\
\text { pointer scaling }\end{array}$ & 468 & Basic & 1782 & \\
\hline Null Dereference & 476 & Basic & 2193 & \\
\hline
\end{tabular}




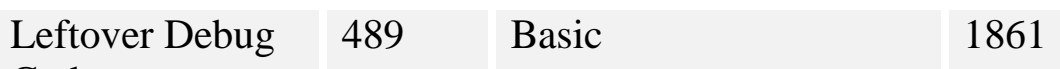

Code

\subsection{Test Suites for the C++ Language}

\subsubsection{Test Suite SCA-TS-1-CPP (SRD Test Suite 57)}

http://samate.nist.gov/SRD/view.php?tsID=47

This test suite tests the capability of a source code security analysis tool's handling weaknesses in the $\mathrm{C}++$ language. Coupled with SCA-TS-1-C (SRD Test Suite 45), it covers the source code weaknesses in $\mathrm{C}++$ listed in Annex A of Source Code Security Analysis Tool Functional Specification [SCA].

\begin{tabular}{|c|c|c|c|c|}
\hline $\begin{array}{l}\text { Source Code } \\
\text { Weakness }\end{array}$ & $\begin{array}{l}\text { CWE } \\
\text { ID }\end{array}$ & Code Complexity & $\begin{array}{l}\text { SRD } \\
\text { Test } \\
\text { case ID }\end{array}$ & Remark \\
\hline \multirow[t]{3}{*}{ Basic XSS } & 80 & Basic & 1965 & \\
\hline & & Scope & 1973 & \\
\hline & & Index alias level & 1975 & \\
\hline \multirow{4}{*}{$\begin{array}{l}\text { Resource } \\
\text { Injection }\end{array}$} & 99 & Basic & 2013 & \\
\hline & & Scope & 2023 & \\
\hline & & Address alias level & 2021 & \\
\hline & & Container & 2026 & \\
\hline \multirow{4}{*}{$\begin{array}{l}\text { OS Command } \\
\text { Injection }\end{array}$} & 78 & Basic & 2028 & \\
\hline & & Scope & 2030 & \\
\hline & & Local control flow & 2032 & \\
\hline & & Loop structure & 2034 & \\
\hline \multirow[t]{3}{*}{ SQL Injection } & 89 & Basic & 1983 & \\
\hline & & Array index complexity & 1989 & \\
\hline & & Scope & 1985 & \\
\hline \multirow[t]{2}{*}{ Stack Overflow } & 121 & Basic & 1971 & \\
\hline & & Container & 2038 & \\
\hline \multirow[t]{4}{*}{ Heap Overflow } & 122 & Basic & 2062 & \\
\hline & & Scope & 2063 & \\
\hline & & $\begin{array}{l}\text { Array address } \\
\text { complexity }\end{array}$ & 2064 & \\
\hline & & Array index complexity & 2065 & \\
\hline Hard-coded & 259 & Basic & 2043 & \\
\hline
\end{tabular}




\begin{tabular}{|l|l|l|l|l|}
\hline Password & & Scope & 2044 & \\
\hline & & Loop structure & 2045 & \\
Container & Data Type & 2046 & \\
\hline & & Basic & 1739 & \\
\hline $\begin{array}{l}\text { Unchecked Error } \\
\text { Condition }\end{array}$ & 391 & Basic & 2054 & \\
\hline Memory leak & 401 & Local Control Flow & 2056 & Data type is integer \\
\hline $\begin{array}{l}\text { Uninitialized } \\
\text { variable }\end{array}$ & 457 & Data type & 2187 & Data type is pointer \\
\hline & & Data type & 2060 & \\
\hline $\begin{array}{l}\text { Unintentional } \\
\text { pointer scaling }\end{array}$ & 468 & Basic & 2188 & \\
\hline $\begin{array}{l}\text { Null Pointer } \\
\text { Dereference }\end{array}$ & 476 & Basic & 1979 & \\
\hline & & Index alias level & 1999 & \\
\hline & & Local control flow & 1997 & \\
\hline $\begin{array}{l}\text { Leftover Debug } \\
\text { Code }\end{array}$ & 489 & Basic & 1995 & \\
\hline
\end{tabular}

\subsubsection{Test Suite SCA-TS-2-CPP (SRD Test Suite 58)}

http://samate.nist.gov/SRD/view.php?tsID=58

This test suite can be used to assess the false positive ratio of the tool under test for C++ applications. Coupled with SCA-TS-2-C (SRD Test Suite 46), it covers the source code weaknesses in C++ listed in Annex A of Source Code Security Analysis Tool Functional Specification [SCA].

\begin{tabular}{|l|l|l|l|l|}
\hline $\begin{array}{l}\text { Source Code } \\
\text { Weakness }\end{array}$ & $\begin{array}{l}\text { CWE } \\
\text { ID }\end{array}$ & Code Complexity & $\begin{array}{l}\text { SRD } \\
\text { Test } \\
\text { case ID }\end{array}$ & Remark \\
\hline Basic XSS & 80 & Basic & 1966 & \\
\hline & & $\begin{array}{l}\text { Scope } \\
\text { Index alias level }\end{array}$ & 1974 & \\
\hline $\begin{array}{l}\text { Resource } \\
\text { Injection }\end{array}$ & 99 & Basic & 2025 & \\
\hline & & Scope & 2024 & \\
\hline
\end{tabular}




\begin{tabular}{|c|c|c|c|c|}
\hline & & Address alias level & 2022 & \\
\hline & & Container & 2040 & \\
\hline \multirow{4}{*}{$\begin{array}{l}\text { OS Command } \\
\text { Injection }\end{array}$} & 78 & Basic & 2029 & \\
\hline & & Scope & 2036 & \\
\hline & & Local control flow & 2033 & \\
\hline & & Loop structure & 2035 & \\
\hline \multirow[t]{3}{*}{ SQL Injection } & 89 & Basic & 1984 & \\
\hline & & Array index complexity & 1990 & \\
\hline & & Scope & 1986 & \\
\hline \multirow[t]{2}{*}{ Stack Overflow } & 121 & Basic & 1972 & \\
\hline & & Container & 2039 & \\
\hline \multirow[t]{3}{*}{ Heap Overflow } & 122 & Basic & 2066 & \\
\hline & & Scope & 2067 & \\
\hline & & $\begin{array}{l}\text { Array address } \\
\text { complexity }\end{array}$ & 2068 & \\
\hline \multirow{5}{*}{$\begin{array}{l}\text { Hard-coded } \\
\text { Password }\end{array}$} & 259 & Basic & 2047 & \\
\hline & & Local control flow & 2050 & \\
\hline & & Loop structure & 2051 & \\
\hline & & Container & 2052 & \\
\hline & & Data Type & 2049 & \\
\hline $\begin{array}{l}\text { Unchecked Error } \\
\text { Condition }\end{array}$ & 391 & Basic & 1992 & \\
\hline Memory leak & 401 & Basic & 2058 & \\
\hline \multirow{3}{*}{$\begin{array}{l}\text { Uninitialized } \\
\text { variable }\end{array}$} & 457 & Data type & 2191 & Data type is integer \\
\hline & & Data type & 2061 & Data type is pointer \\
\hline & & Loop Structure & 2192 & \\
\hline $\begin{array}{l}\text { Unintentional } \\
\text { Pointer Scaling }\end{array}$ & 468 & Basic & 1980 & \\
\hline \multirow{4}{*}{$\begin{array}{l}\text { Null Pointer } \\
\text { Dereference }\end{array}$} & 476 & Basic & 1994 & \\
\hline & & Index alias level & 2000 & \\
\hline & & Local control flow & 1998 & \\
\hline & & Scope & 1996 & \\
\hline $\begin{array}{l}\text { Leftover Debug } \\
\text { Code }\end{array}$ & 489 & Basic & 2197 & \\
\hline
\end{tabular}




\subsubsection{Test Suite SCA-TS-3-CPP (SRD Test Suite 59)}

http://samate.nist.gov/SRD/view.php?tsID=59

This test suite can be used to examine whether the tool generates weakness reports after reporting of each weakness has been suppressed for $\mathrm{C}++$ applications. Coupled with SCA-TS-3-C (SRD Test Suite 47), it covers the source code weaknesses in C++ listed in Annex A of Source Code Security Analysis Tool Functional Specification [SCA].

\begin{tabular}{|c|c|c|c|c|}
\hline $\begin{array}{l}\text { Source Code } \\
\text { Weakness }\end{array}$ & $\begin{array}{l}\text { CWE } \\
\text { ID }\end{array}$ & Code Complexity & $\begin{array}{l}\text { SRD } \\
\text { Test } \\
\text { case ID }\end{array}$ & Remark \\
\hline Basic XSS & 80 & Basic & 1965 & \\
\hline $\begin{array}{l}\text { Resource } \\
\text { Injection }\end{array}$ & 99 & Basic & 2013 & \\
\hline $\begin{array}{l}\text { OS Command } \\
\text { Injection }\end{array}$ & 78 & Basic & 2028 & \\
\hline SQL Injection & 89 & Basic & 1983 & \\
\hline Stack Overflow & 121 & Basic & 1971 & \\
\hline Heap Overflow & 122 & Basic & 2062 & \\
\hline $\begin{array}{l}\text { Hard-coded } \\
\text { Password }\end{array}$ & 259 & Basic & 2043 & \\
\hline $\begin{array}{l}\text { Unchecked Error } \\
\text { Condition }\end{array}$ & 391 & Basic & 1739 & \\
\hline Memory leak & 401 & Basic & 2054 & \\
\hline $\begin{array}{l}\text { Uninitialized } \\
\text { variable }\end{array}$ & 457 & Data type & 2187 & Data type is integer \\
\hline $\begin{array}{l}\text { Unintentional } \\
\text { pointer scaling }\end{array}$ & 468 & Basic & 1979 & \\
\hline $\begin{array}{l}\text { Null Pointer } \\
\text { Dereference }\end{array}$ & 476 & Basic & 1993 & \\
\hline $\begin{array}{l}\text { Leftover Debug } \\
\text { Code }\end{array}$ & 489 & Basic & 2196 & \\
\hline
\end{tabular}

\subsection{Test Suites for the Java Language}

\subsubsection{Test Suite SCA-TS-1-JAVA (SRD Test Suite 63)} http://samate.nist.gov/SRD/view.php?tsID=63 
This test suite tests the capability of a source code security analysis tool's handling of weaknesses in the Java language. It covers the source code weaknesses in Java listed in Annex A of Source Code Security Analysis Tool Functional Specification [SCA].

\begin{tabular}{|c|c|c|c|c|}
\hline $\begin{array}{l}\text { Source Code } \\
\text { Weakness }\end{array}$ & $\begin{array}{l}\text { CWE } \\
\text { ID }\end{array}$ & Code Complexity & $\begin{array}{l}\text { SRD } \\
\text { Test } \\
\text { case ID }\end{array}$ & Remark \\
\hline \multirow[t]{4}{*}{ Basic XSS } & 80 & Basic & 2153 & \\
\hline & & Scope & 2154 & \\
\hline & & Container & 2155 & \\
\hline & & Loop complexity & 2156 & \\
\hline \multirow{3}{*}{$\begin{array}{l}\text { Resource } \\
\text { Injection }\end{array}$} & 99 & Basic & 2088 & \\
\hline & & Scope & 2089 & \\
\hline & & Container & 2090 & \\
\hline \multirow{4}{*}{$\begin{array}{l}\text { OS Command } \\
\text { Injection }\end{array}$} & 78 & Basic & 2084 & \\
\hline & & Scope & 2085 & \\
\hline & & Local control flow & 2086 & \\
\hline & & Loop structure & 2087 & \\
\hline \multirow[t]{3}{*}{ SQL Injection } & 89 & Basic & 2161 & \\
\hline & & Array index complexity & 2162 & \\
\hline & & Scope & 2163 & \\
\hline \multirow{5}{*}{$\begin{array}{l}\text { Hard-coded } \\
\text { Password }\end{array}$} & 259 & Basic & 2091 & \\
\hline & & Local control flow & 2092 & \\
\hline & & Loop structure & 2093 & \\
\hline & & Container & 2094 & \\
\hline & & Array Index Complexity & 2095 & \\
\hline $\begin{array}{l}\text { Time-of-check } \\
\text { Time-of-use race } \\
\text { condition }\end{array}$ & 367 & Basic & 2096 & \\
\hline $\begin{array}{l}\text { Unchecked Error } \\
\text { Condition }\end{array}$ & 391 & Basic & 2103 & \\
\hline $\begin{array}{l}\text { Unrestricted } \\
\text { Critical Resource } \\
\text { Lock }\end{array}$ & 412 & Basic & 2104 & \\
\hline \multirow[t]{4}{*}{ Null Dereference } & 476 & Basic & 2099 & \\
\hline & & Address alias level & 2105 & \\
\hline & & Local control flow & 2106 & \\
\hline & & Scope & 2107 & \\
\hline
\end{tabular}


\begin{tabular}{l|l|l|l}
$\begin{array}{l}\text { Leftover Debug } \\
\text { Code }\end{array}$ & 489 & Basic & 2098
\end{tabular}

\subsubsection{Test Suite SCA-TS-2-JAVA (SRD Test Suite 64)}

http://samate.nist.gov/SRD/view.php?tsID=64

This test suite can be used to assess the false positive ratio of the tool under test for JAVA applications.

\begin{tabular}{|c|c|c|c|c|}
\hline $\begin{array}{l}\text { Source Code } \\
\text { Weakness }\end{array}$ & $\begin{array}{l}\text { CWE } \\
\text { ID }\end{array}$ & Code Complexity & $\begin{array}{l}\text { SRD } \\
\text { Test } \\
\text { case ID }\end{array}$ & Remark \\
\hline \multirow[t]{4}{*}{ Basic XSS } & 80 & Basic & 2157 & \\
\hline & & Scope & 2158 & \\
\hline & & Container & 2159 & \\
\hline & & Loop complexity & 2160 & \\
\hline \multirow{3}{*}{$\begin{array}{l}\text { Resource } \\
\text { Injection }\end{array}$} & 99 & Basic & 2114 & \\
\hline & & Scope & 2115 & \\
\hline & & Container & 2116 & \\
\hline \multirow{4}{*}{$\begin{array}{l}\text { OS Command } \\
\text { Injection }\end{array}$} & 78 & Basic & 2110 & \\
\hline & & Scope & 2111 & \\
\hline & & Local control flow & 2112 & \\
\hline & & Loop structure & 2113 & \\
\hline \multirow[t]{3}{*}{ SQL Injection } & 89 & Basic & 2164 & \\
\hline & & Array index complexity & 2165 & \\
\hline & & Scope & 2166 & \\
\hline \multirow{5}{*}{$\begin{array}{l}\text { Hard-coded } \\
\text { Password }\end{array}$} & 259 & Basic & 2117 & \\
\hline & & Local control flow & 2118 & \\
\hline & & Loop structure & 2119 & \\
\hline & & Container & 2120 & \\
\hline & & Array Index Complexity & 2121 & \\
\hline $\begin{array}{l}\text { Time-of-check } \\
\text { Time-of-use race } \\
\text { condition }\end{array}$ & 367 & Basic & 2122 & \\
\hline $\begin{array}{l}\text { Unchecked Error } \\
\text { Condition }\end{array}$ & 391 & Basic & 2123 & \\
\hline Unrestricted & 412 & Basic & 2124 & \\
\hline
\end{tabular}




\begin{tabular}{|l|l|l|l|}
\hline $\begin{array}{l}\text { Critical Resource } \\
\text { Lock }\end{array}$ & & & \\
\hline Null Dereference & 476 & Basic & 2125 \\
\hline & & Address alias level & 2128 \\
\hline & & Local control flow & 2126 \\
\hline & & Scope & 2127 \\
\hline $\begin{array}{l}\text { Leftover Debug } \\
\text { Code }\end{array}$ & 489 & Basic & 2129 \\
\hline
\end{tabular}

\subsubsection{Test Suite SCA-TS-3-JAVA (SRD Test Suite 65)}

http://samate.nist.gov/SRD/view.php?tsID=65

This test suite can be used to examine whether the tool generates weakness reports after the reporting of each weakness has been suppressed for JAVA applications.

\begin{tabular}{|c|c|c|c|c|}
\hline $\begin{array}{l}\text { Source Code } \\
\text { Weakness }\end{array}$ & $\begin{array}{l}\text { CWE } \\
\text { ID }\end{array}$ & Code Complexity & $\begin{array}{l}\text { SRD } \\
\text { Test } \\
\text { case ID }\end{array}$ & Remark \\
\hline Basic XSS & 80 & Basic & 2153 & \\
\hline $\begin{array}{l}\text { Resource } \\
\text { Injection }\end{array}$ & 99 & Basic & 2088 & \\
\hline $\begin{array}{l}\text { OS Command } \\
\text { Injection }\end{array}$ & 78 & Basic & 2084 & \\
\hline SQL Injection & 89 & Basic & 2161 & \\
\hline $\begin{array}{l}\text { Hard-coded } \\
\text { Password }\end{array}$ & 259 & Basic & 2091 & \\
\hline $\begin{array}{l}\text { Time-of-check } \\
\text { Time-of-use race } \\
\text { condition }\end{array}$ & 367 & Basic & 2096 & \\
\hline $\begin{array}{l}\text { Unchecked Error } \\
\text { Condition }\end{array}$ & 391 & Basic & 2103 & \\
\hline $\begin{array}{l}\text { Unrestricted } \\
\text { Critical Resource } \\
\text { Lock }\end{array}$ & 412 & Basic & 2104 & \\
\hline Null Dereference & 476 & Basic & 2099 & \\
\hline $\begin{array}{l}\text { Leftover Debug } \\
\text { Code }\end{array}$ & 489 & Basic & 2098 & \\
\hline
\end{tabular}




\section{References}

[CWE] Common Weakness Enumeration - a community-developed dictionary of software weakness types, sponsored and managed by The MITRE

Corporation.

http://cwe.mitre.org/

[Fleiss] Fleiss, Joseph L. (1981). Statistical Methods for Rates and Proportions, 2nd ed., John Wiley and Sons, New York, pp 4-8.

[SCA] Source Code Security Analysis Tool Functional Specification Version 1.0. http://samate.nist.gov/docs/source_code_security_analysis_spec_SP500268.pdf

[SRD] SAMATE Reference Dataset

http://samate.nist.gov/SRD/

To retrieve test suite, click on "Test Suites" on the tool bar. A list of test suite will display. Select the required test suite. 